\title{
Identification and diversity of Fusarium species isolated from tomato fruits
}

\author{
Nur Baiti Abd Murad, Nor Azizah Kusai, Nur Ain Izzati Mohd Zainudin* \\ Department of Biology, Faculty of Science, Universiti Putra Malaysia, 43400 UPM Serdang, Selangor, Malaysia
}

Received: January 20, 2016

Accepted: June 20, 2016

\begin{abstract}
Fruit rot of tomato is a serious disease caused by Fusarium species. Sampling was conducted throughout Selangor, Malaysia and fungal species identification was conducted based on morphological and gene encoding translation elongation factor 1- $\alpha$ (tef1- $\alpha$ ) sequence analysis. Five species of Fusarium were discovered namely F. oxysporum (including F. oxysporum f. sp. lycopersici), F. solani, F. equiseti, F. proliferatum and F. verticillioides. Our results provide additional information regarding the diversity of Fusarium species associated with fruit rot disease of tomato.
\end{abstract}

Key words: diversity, fruit rot, Fusarium oxysporum, Gibberella fujikuroi, translation elongation factor

\section{Introduction}

Tomato (Lycopersicon esculentum Mill.) contains many nutrients for human health such as dietary lycopene, $\beta$-carotene, carotenoids, vitamins $\mathrm{C}$ and $\mathrm{E}$, potassium, fibre and antioxidant elements (Britt and Kristin 2011; Troncoso-Rojas et al. 2013). However, tomato plants are often infected by field and storage rot disease pathogens. There are several factors leading to these diseases such as poor packaging and improper management. These factors can cause the fruit at the market to wilt and squash easily (Taskeen-Un-Nisa et al. 2011; Xie et al. 2012). According to Kristensen et al. (2005), the most widely distributed pathogen of tomato fruit rot is Fusarium species. Fusarium species can produce mycotoxins such as moniliformin, fumonisins $\mathrm{B}_{1}$, zearalenone, beuvericin and trichothecenes (Wiśniewska et al. 2011; Zainudin et al. 2015), which are important issues for human health.

Abu Bakar et al. (2013) reported that there are five Fusarium species, which have only been tentatively identified based on morphological characteristics. They also identified the causal agents of fruit rot of tomato in Malaysia that include Fusarium semitectum, F. oxysporum, F. equiseti, F. subglutinans and F. solani. Realising that information about the diversity of Fusarium species associated with fruit rot of tomato based on gene sequencing is limited in tropical areas we have taken the initiative to characterise some isolates of Fusarium species based on gene encoding translation elongation factor $1-\alpha$ (tef1- $\alpha$ ) sequence. Translation elongation factor $1-\alpha$ (tef1- $\alpha$ ) is an important part of protein translation machinery, which is a good single locus identification tool in Fusarium because it shows high sequence polymorphism among closely related species (Nitschke et al. 2009; Amatulli et al. 2010; Walker et al. 2012).
The correct identification of species associated with post-harvest fruit rot of tomato will affect the country's quarantine regulations. Therefore, the aim of this study was to characterise the Fusarium strains associated with post-harvest fruit rot of tomato based on morphological characteristics and tef1- $\alpha$ gene sequence analysis.

\section{Materials and Methods}

\section{Sampling and fungal isolation}

Fruit rot of tomato was observed and the tomato samples were obtained from markets throughout Selangor, Malaysia. Ten tomato samples showing fruit rot symptoms were collected from different individual markets. The symptoms observed were similar to those reported in previous literature, namely the rotted tissue was often water-soaked and covered by white to pinkish mycelia externally while the infected tissue was found to be discoloured and watery. Samples, $5 \times 5 \mathrm{~mm}$ from the border of symptomatic and asymptomatic tissue, were cut. The surface of diseased samples was sterilized with $1 \%$ sodium hypochlorite. Samples were then rinsed in sterile distilled water. All disinfected samples were placed onto peptone pentachloronitrobenze agar (PPA) and incubated at $28^{\circ} \mathrm{C}$ for five days.

\section{Fungal purification and morphological characterization}

The fungal cultures were transferred to Water Agar (WA) for single conidial isolation. The conidial suspensions of the cultures were streaked into $4 \%$ WA, which was then incubated for $24 \mathrm{~h}$. A hyphal tip derived from a single colony was cut and transferred onto new Potato Dextrose 
Agar (PDA), and then incubated for 7 days. Pure cultures of Fusarium isolates were subcultured and maintained as a conidial suspension in $25 \%$ glycerol of Complete Medium with Xylose $(\mathrm{CMX})$ at $-80^{\circ} \mathrm{C}$.

All purified isolates were tentatively identified based on morphological characteristics. The isolates were cultured on PDA and incubated at $25^{\circ} \mathrm{C}$ with $12 \mathrm{~h}$ fluorescent light/12 h darkness for 7 days. Macroscopic characteristics, which were observed included colony features, color and growth rate. The pure isolates were also cultured on Carnation Leaf Agar (CLA). Microscopic characteristics such as conidia shape and size were examined using a microscope (Carl Zeiss, Germany) (Leslie and Summerell 2006).

\section{DNA extraction and polymerase chain reaction (PCR) amplification}

DNA extraction was performed using the Ultra Clean ${ }^{\circledR}$ Microbial DNA isolation kit (MO-BIO, Carlsbad, CA, USA) according to the manufacturer's instructions. The PCR amplification of tef1- $\alpha$ was performed using a TProfessional Standard Thermocycler (Biometra Company); $25 \mu \mathrm{l}$ reaction master mix that contained $5 \times$ PCR buffer, $0.5 \mu \mathrm{M}$ of primer, $0.2 \mathrm{mM}$ dNTPs, $2.5 \mathrm{mM}$ magnesium chloride $\left(\mathrm{MgCl}_{2}\right), 0.125$ unit of Taq Polymerase (Promega Corporation, 2012) and 20 ng of DNA template. A set of primers was used; EF1 (5'-ATGGGTAAGGAGGACAAGAC-3') and EF2 (5'-GGAAGTACCAGTGATCATGTT-3') to amplify $700 \mathrm{bp}$ fragment (Geiser et al. 2004). The PCR cycling was conducted as follows: initial denaturation at $94^{\circ} \mathrm{C}$ for $85 \mathrm{~s}$, followed by 35 cycles of denaturation at $95^{\circ} \mathrm{C}$ for $35 \mathrm{~s}$, annealing at $57^{\circ} \mathrm{C}$ for $55 \mathrm{~s}$, extension at $72^{\circ} \mathrm{C}$ for $90 \mathrm{~s}$, final extension at $72^{\circ} \mathrm{C}$ for $10 \mathrm{~min}$.

\section{Nucleotide sequencing and analysis}

Products of amplification were electrophoresed while the gels were stained with ethidium bromide $(\mathrm{EtBr})$ and the DNA bands were visualised under ultraviolet (UV) light. The images were captured with DOC PRINT system (Vilber Lourmat, USA). Target DNA fragment band with sized 700 bp (Nitschke et al. 2009) was excised from the gel, followed by the purification of PCR products using QIAquick ${ }^{\circledR}$ Gel Extraction Kit (QIAGEN, USA) following the manufacturer's recommendations.

The purified PCR products were sequenced for the tef1- $\alpha$ gene region using ABI3730XL sequencer (MyTACG Bioscience Company, MY). The comparison between tef1- $\alpha$ gene sequence of analysed isolates and the sequences in GenBank was done using the Standard Nucleotide BLAST network services for similarities present in the National Centre for Biotechnology Information (NCBI) database (http://www.ncbi.nlm.nih.gov/). ClustalW in MEGA software version 6.06 was used to generate the consensus sequences for alignment (Tamura et al. 2013).

A phylogenetic tree was also generated using MEGA software analysis version 6.06. Maximum likelihood (ML) analysis was performed and the tree was built with Tamura-Nei model. One thousand bootstrap replicates were performed to assess the stability and robustness of each branch of the phylogenetic tree (Tamura et al. 2013).
All assembled sequences were then deposited to GeneBank, NCBI (http://www.ncbi.nlm.nih.gov/). A tef1- $\alpha$ sequence of Colletotrichum acutatum (JQ958570) from GenBank was used as an out-group and sequences of tef1- $\alpha$ gene of Fusarium spp. from NCBI were used as references for the strains identified in this study.

\section{Results}

\section{Morphological characteristics}

Based on morphological characteristics, the most important features of F. oxysporum are the formation of macroconidia with short-medium length, thick-walled, curved dorsal curvature and a slightly straighter ventral surface, tapered and curved with hook apical cells and footshaped basal cells. Microconidia are oval to reniformshaped and frequently produced in alse heads mycelia on short monophialides. Other characteristics considered are white to pale violet of abundant mycelia and white tinged with purple pigments in the agar. Meanwhile, chlamydospores are oval, which were formed singly and in pairs terminally and intercalary with smooth and rough walls. Macroconidia were observed to obtain 3-5 septa, while microconidia have only $0-1$ septum. No difference in morphological characteristics was observed among F. oxysporum races.

The most conspicuous microscopic feature of $F$. solani is its ability to produce $0-1$ septum oval to reniformshaped microconidia in false heads mycelia that usually are borne on long monophialides. Macroconidia were found with 3-5 septa, having apical cells that are curved and rounded as well as foot-shaped basal cells. The colonies on PDA were white to cream coloured with sparse mycelia, which produced cream to light yellow pigments. Chlamydospores were found to be singly, intercalary and terminal.

Fusarium proliferatum produced thin-walled, slender and rather straight macroconidia. The apical cell was curved and usually formed with 3-5 septates. Microconidia were oval to obovoid-shaped and arose in false heads and moderate length chains. They were derived from monophialides and also polyphialides conidiogenous cells. Chlamydospores were absent in the culture of F. proliferatum. On PDA, F. proliferatum initially produced white aerial mycelia and became cottony with a light orange pigment.

Macroconidia of F. verticillioides were slender, slightly straight, thin-walled and typically formed with three septates. Their apical cell was blunted and barely notched on the basal cell. The microconidia were oval to obovoidshaped. Compared with cultures of F. proliferatum, F. verticillioides produced longer chains of microconidia, which also arose in false heads. Furthermore, the culture of F. verticillioides generated microconidia that were only derived from monophialides conidiogenous cells. No polyphialides were produced by F. verticillioides. Fusarium verticillioides culture did not produce chlamydospores. However, they produced swollen cells that can easily be misidentified as chlamydospores. The cultures were cottony flat, which initially grew as white mycelial growth 
and covered the PDA plate after nine days of incubation, with orange pigmentation.

The cultures of $F$. equiseti produced long macroconidia, thick-walled, sickle-shaped and curved dorsiventral curvature, tapered, whip-like apical cells and elongated footshaped basal cells. Meanwhile, the colonies produced abundant white and brown mycelia and pale brown pigments on PDA. Chlamydospores were found to be oval and formed singly, in pairs, in chains or in clumps. Furthermore, microconidia were formed in false heads.

\section{Molecular characteristics, sequencing and phylogenetic analysis}

Species identification of Fusarium species was confirmed by the sequences of tef1- $\alpha$. Results showed a clear single band that was amplified and corresponded to the expected molecular fragment size of the tef1- $\alpha$ region. No band was observed in the control, where DNA was replaced with water. These PCR amplification results made it possible to predict that all tested strains belonged to the genus Fusarium.

The Standard Nucleotide BLAST search for similarities showed that the similarity percentage of the strains ranged from 98 to $99 \%$. The tef1- $\alpha$ sequence of all $\mathrm{Fu}$ sarium strains were searched for homology sequences in GeneBank database. The similarities of tef1- $\alpha$ sequence of our strains with the sequences from the GeneBank used as references were supported by bootstrap values of more than $70 \%$ as stated in this study. All the analysed sequences data were deposited in the GeneBank database (Table 1). The phylogenetic tree was divided into

Table 1. List of Fusarium strains isolated from post-harvest fruit rot of tomato in Selangor, Malaysia analysed based on tef1- $\alpha$ gene sequence and pathogenicity test

\begin{tabular}{|c|c|c|c|c|}
\hline No. & Strains number & Locations & $\begin{array}{c}\text { GeneBank } \\
\text { accession number }\end{array}$ & $\begin{array}{l}\text { Species identification based } \\
\text { on tef1- } \alpha \text { gene sequence }\end{array}$ \\
\hline 1 & B616T & Serdang & KM886217 & F. solani \\
\hline 2 & B627T & Serdang & KM886223 & F. solani \\
\hline 3 & B664T & Serdang & KM886218 & F. solani \\
\hline 4 & $\mathrm{~B} 780 \mathrm{~T}$ & Sungai Buloh & KM886219 & F. solani \\
\hline 5 & $\mathrm{~B} 684 \mathrm{~T}$ & Kajang & KM886224 & F. solani \\
\hline 6 & $\mathrm{~B} 698 \mathrm{~T}$ & Kajang & KM886220 & F. solani \\
\hline 7 & B777T & Sungai Buloh & KM886221 & F. solani \\
\hline 8 & $\mathrm{~B} 722 \mathrm{~T}$ & Semenyih & KM886222 & F. solani \\
\hline 9 & $\mathrm{~B} 702 \mathrm{~T}$ & Kajang & KM886226 & F. solani \\
\hline 10 & $\mathrm{~B} 752 \mathrm{~T}$ & Bandar Sunway & KM886227 & F. solani \\
\hline 11 & B1349T & Serdang & KM886212 & F. equiseti \\
\hline 12 & B1350T & Serdang & KM886225 & F. solani \\
\hline 13 & B1351T & Ampang & KM886229 & F. solani \\
\hline 14 & B1352T & Ampang & KM886230 & F. solani \\
\hline 15 & B1353T & Ampang & KM886231 & F. solani \\
\hline 16 & B1354T & Kajang & KM886232 & F. solani \\
\hline 17 & B1355T & Ampang & KM886233 & F. solani \\
\hline 18 & B1356T & Kajang & KM886228 & F. solani \\
\hline 19 & B633T & Serdang & KM886238 & F. oxysporum f. sp. lycopersici \\
\hline 20 & B645T & Serdang & KM886239 & F. oxysporum f. sp. lycopersici \\
\hline 21 & B688T & Kajang & KM873335 & F. oxysporum \\
\hline 22 & B696T & Kajang & KM873336 & F. oxysporum \\
\hline 23 & B711T & Kajang & KM886241 & F. oxysporum f. sp. lycopersici \\
\hline 24 & B713T & Kajang & KM886240 & F. oxysporum f. sp. lycopersici \\
\hline 25 & B717T & Semenyih & KM886236 & F. oxysporum f. sp. lycopersici \\
\hline 26 & B718T & Semenyih & KM886237 & F. oxysporum f. sp. lycopersici \\
\hline 27 & B725T & Puchong & KM886213 & F. oxysporum \\
\hline 28 & B635T & Serdang & KM873337 & F. oxysporum \\
\hline 29 & $\mathrm{~B} 622 \mathrm{~T}$ & Serdang & KM886215 & F. oxysporum \\
\hline 30 & B695T & Serdang & KM886216 & F. oxysporum \\
\hline 31 & B1358T & Selayang & KM886214 & F. oxysporum \\
\hline 32 & B1357T & Serdang & KM873334 & F. proliferatum \\
\hline 33 & B1359T & Selayang & KM886234 & F. verticillioides \\
\hline 34 & B1360T & Selayang & KM886235 & F. verticillioides \\
\hline
\end{tabular}




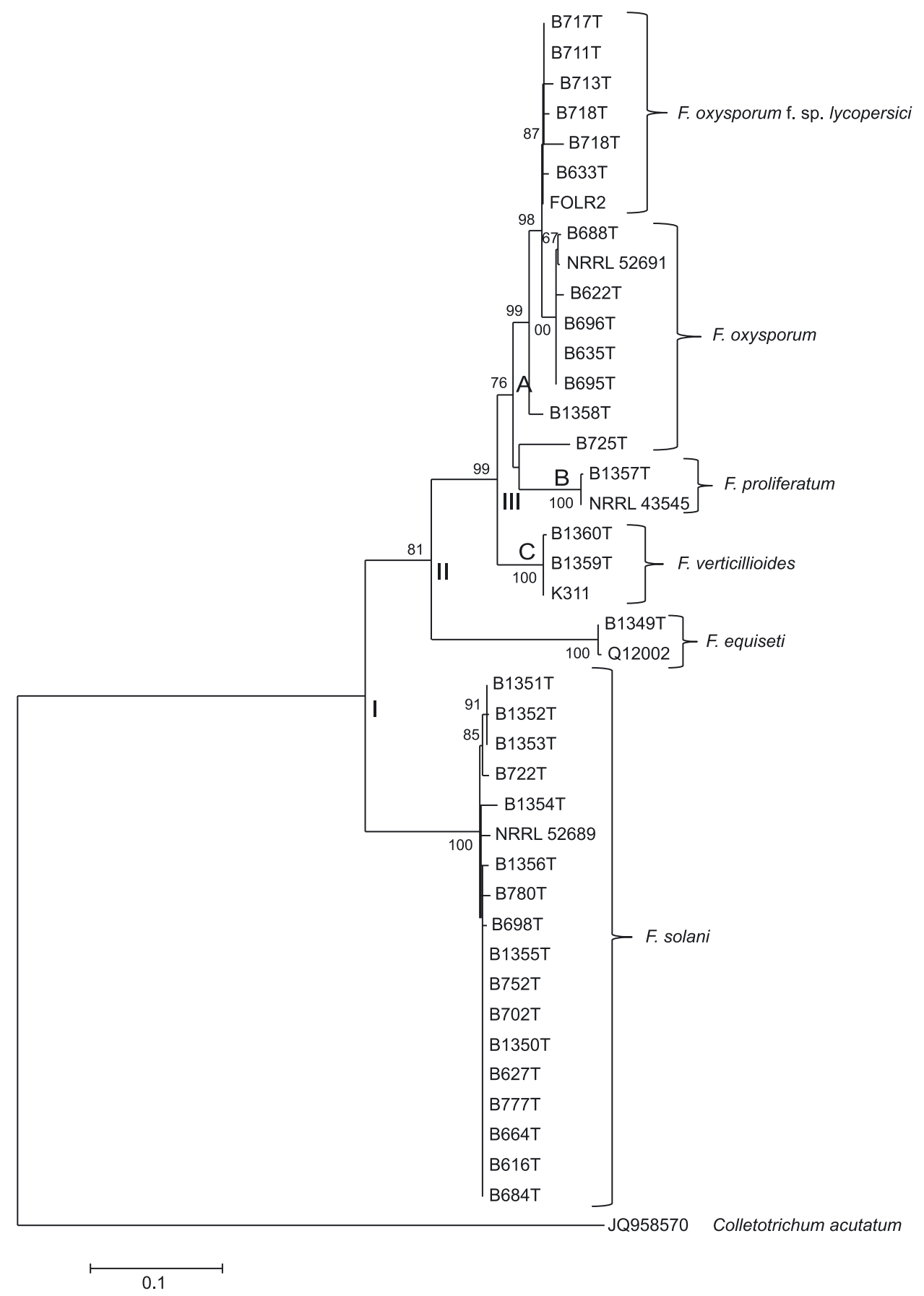

Fig. 1. Phylogenetic tree showing the relationship of 34 Fusarium species strains based on tef1- $\alpha$ gene sequence using the maximum likelihood method. The percentage of replicate trees in which the linked taxa clustered together in the bootstrap test $(1,000$ replicates). The bootstraps values $>70 \%$ were shown next to the branches. Accession numbers of FOLR2 (DQ837692), NRRL 52691 (JF740776), NRRL 43545 (EF452971), K311 (KF562131), Q12002 (KF208617) and NRRL 52689 (JF740774). Colletotrichum acutatum was used as an out-group. I, II, III - clades; A, B, C - subclades

three main clades, known as I, II and III (Fig. 1). Clade I shows that all the strains demonstrated high similarities of tef1- $\alpha$ gene sequence to each other and to the referred F. solani (NRRL52689). All strains were well supported by the strongest bootstrap value of $100 \%$. On the other hand, Clade II that consists of only single strain B1349T also gave high similarity of tef1- $\alpha$ gene sequence to the referred F. equiseti (Q12002) from NCBI and was supported by the strongest bootstrap value of $100 \%$. Furthermore, Clade III contains three subclades of A, B and C. Strains grouped in subclade A have high similarities of tef1- $\alpha$ gene sequence to each other and to the control strains of F. oxysporum (NRRL52691) and F. oxysporum f. sp. lycopersici (FOLR2). In this analysis, subclade A was supported by the bootstrap value of $76 \%$. This also includes six strains namely B713T, B711T, B718T, B717T, B645T and B633T that belong to the formae speciales of lycopersici supported by the bootstrap value of $87 \%$. Subclade B contains only a single strain B1357T, which also gave high similarity of tef1- $\alpha$ sequence to the referred $F$. proliferatum (NRRL43545) that was supported by the strongest bootstrap value of $100 \%$. Morphology characters confirmed the species identity 
of isolate B725T since it was grouped separately from other isolates of F. oxysporum. Moreover, subclade $C$ that comprises two strains, B1360T and B1359T also gave the strongest bootstrap value of $100 \%$, which referred with F. verticillioides (K311).

\section{Discussion}

In the present study, a combination of morphology and tef1- $\alpha$ gene sequencing were used to identify the isolates into species levels. Six isolates were identified into formae speciales based on tef1- $\alpha$ gene sequence. There are limits to the use of morphological characters for identification of species in Gibberella fujikuroi species complex and F. oxysporum as some species may share similar morphological characteristics, for example F. proliferatum and F. verticillioides. This study provides an update regarding the diversity of Fusarium species associated with fruit rot of tomato. Abu Bakar et al. (2013) identified five species of Fusarium associated with fruit rot disease of tomato in Malaysia, which are F. oxysporum, F. solani, F. semitectum, F. subglutinans and F. equiseti. However, in this study, we isolated and identified two other species: F. proliferatum and $F$. verticillioides.

Based on morphological characteristics, F. proliferatum is most likely to be confused with $F$. verticillioides where they may be found in chains of varying length (Leslie and Summerell 2006). Moreover, relying only on the presence of polyphialides is not rigid enough evidence to differentiate $F$. proliferatum and F. verticillioides. Misidentification between these two species of Fusarium often occurs since they have similar pigmentation, shape of macroconidia and microconidia and the formation of a false head. Thus, a molecular method, was used to differentiate DNA sequences of gene, to support morphological identification between F. proliferatum and F. verticillioides. This also happens in the identification of $F$. oxysporum isolates into formae speciales. Many F. oxysporum isolates appear to be host specific (Leslie and Summerell 2006). Over 100 formae speciales and races of F. oxysporum have been described. In this study, we found six isolates which belonged to F. oxysporum f. sp. lycopersici.

Based on the generated phylogenetic tree in this study, F. oxysporum including F. oxysporum f. sp. lycopersici, F. verticillioides and $F$. proliferatum were grouped in Clade III, which was named the Gibberella clade that further divided into two different sections, where, F. oxysporum was located in section Elegans, while the F. verticillioides and F. proliferatum were located in section Liseola. They were then grouped into different subclades as A, B, and $C$, respectively. For Clade I and II, F. equiseti and F. solani have singly occurred without any subclade grouping. However, the subclades grouping in Clade III showed that the F. oxysporum, F. verticillioides and F. proliferatum are not closely related to each other since they are from a different Fusarium species complex. Fusarium oxysporum is a member of the F. oxysporum species complex, while F. verticillioides and F. proliferatum are members of the Gibberella fujikuroi species complex (GFC) (Kvas et al. 2009). Even though $F$. verticillioides and F. proliferatum are in the same section of Liseola, based on the generated tree, they were formed by two separated subclades. The GFC is made-up of three different clades known as Asian, American and African clades. On the other hand, F. verticillioides is from the African clade, while F. proliferatum is from the Asian clade as stated in the previous study conducted by Kvas et al. (2009).

In addition, Geiser et al. (2004) also classified F. verticillioides and F. proliferatum as GFC, while the other Fusarium species as the member of respective species complex where F. oxysporum is F. oxysporum and relatives' species complex, F. solani is a member of the F. solani species complex and F. equiseti is a member of the F. equiseti species complex. These findings are consistent with the previous study conducted by Watanabe et al. (2011), which reported that F. oxysporum, F. verticillioides and F. proliferatum are in the same clade (Gibberella), but in different sections of Elegans and Liseola, respectively. Whereas F. solani and F. equiseti are in two other clades as well as in a different section of Martiella and Ventricosum, and Gibbosum when referring to their ML tree of Fusarium genus and related genera inferred from the combined sequences of rDNA cluster and the $\beta$-tub and tef1- $\alpha$ genes. Many taxonomic studies based on morphological characteristics have reported that some "sections" including closely related species share some "synapomorphic" character states. Nitschke et al. (2009) also stated in the resulting dendrogram of their study that the F. oxysporum, F. proliferatum and F. verticillioides are grouped in the same cluster or clade.

As for the F. solani and F. equiseti, they are totally unrelated to the other three Fusarium species in Clade III since they are clearly located in different clades, which are Clade I (section Martiella and Ventricosum) and II (section Gibbosum), respectively. Furthermore, both Fusarium species are from different species complexes, where F. solani is a member of $F$. solani species complex, while F. equiseti is a member of other lineages of the F. equiset $i$ species complex.

\section{Acknowledgements}

We thank Mrs. Nor Hidayah Husain for technical assistance. This work was partially supported by Research University Grant Scheme (RUGS) to Nur Ain Izzati Mohd Zainudin. Nur Baiti Abd Murad and Nor Azizah Kusai have received scholarship from the Graduate Research Found of University Putra Malaysia (UPM).

\section{References}

Abu Bakar A.I., Nur Ain Izzati M.Z., Umi Kalsom Y. 2013. Diversity of Fusarium species associated with post-harvest fruit rot disease of tomato. Sains Malaysiana 42 (7): 911-920.

Amatulli M.T., Spadaro D., Gullino M.L., Garibaldi A. 2010. Molecular identification of Fusarium spp. associated with bakanae disease of rice in Italy and assessment of their pathogenicity. Plant Pathology 59 (5): 839-844.

Britt B.F., Kristin R. 2011. Tomato consumption and health: Emerging benefits. American Journal of Lifestyle Medicine 5 (2): 182-191. 
Geiser D.M., Jiménez-Gasco M.M., Kang S., Makalowska I., Veeraraghavan N., Ward T.J., Zhang N., Kuldau G.A., O'Donnell K. 2004. FUSARIUM-ID v. 1.0: A DNA sequence database for identifying Fusarium. European Journal of Plant Pathology 110 (5): 473-479.

Kristensen R., Torp M., Kosiak B., Holst-Jensen A. 2005. Phylogeny and toxigenic potential is correlated in Fusarium species as revealed by partial translation elongation factor 1 alpha gene sequences. Mycological Research 109 (2): 173-186.

Kvas M., Marasas W.F.O., Wingfield B.D., Wingfield M.J., Steenkamp E.T. 2009. Diversity and evolution of Fusarium species in the Gibberella fujikuroi complex. Fungal Diversity 34: $1-21$.

Leslie J.F., Summerell B.A. 2006. The Fusarium Laboratory Manual. 1st ed. Blackwell Publishing Professional, Iowa, USA, $388 \mathrm{pp}$.

Nitschke E., Nihlgard M., Varrelmann M. 2009. Differentiation of eleven Fusarium spp. isolated from sugar beet, using restriction fragment analysis of a polymerase chain reaction amplified translation elongation factor $1 \alpha$ gene fragment. Phytopathology 99 (8): 921-929.

Tamura K., Stecher G., Peterson D., Filipski A., Kumar S. 2013. MEGA6: Molecular evolutionary genetics analysis version 6.0. Molecular Biology and Evolution 30 (12): 2725-2729.

Taskeen-Un-Nisa, Wani A.H., Bhat M.Y., Pala S.A., Mir R.A. 2011. In vitro inhibitory effect of fungicides and botanicals on mycelial growth and spore germination of Fusarium oxysporum. Journal of Biopesticides 4 (1): 53-56.
Troncoso-Rojas R., Sánchez-Estrada A., Carvallo T., González-León A., Ojeda-Contreras J., Aguilar-Valenzuela A., Tiznado-Hernández M. 2013. A fungal elicitor enhances the resistances of tomato fruit to Fusarium oxysporum infection by activating the phenylpropanoid metabolic pathway. Phytoparasitica 41 (2): 133-142.

Walker D.M., Castlebury L.A., Rossman A.Y., White J.F. 2012. New molecular markers for fungal phylogenetics: Two genes for species-level systematics in the Sordariomycetes (Ascomycota). Molecular Phylogenetics and Evolution 64 (3): 500-512.

Watanabe M., Yonezawa T., Lee K., Kumagai S., Sugita-Konishi Y., Goto K., Hara-Kuda Y. 2011. Molecular phylogeny of the higher and lower taxonomy of the Fusarium genus and differences in the evolutionary histories of multiple genes. BMC Evolutionary Biology 11 (1): 322-338.

Wiśniewska H., Basiński T., Chełkowski J., Perkowski J. 2011. Fusarium sporotrichioides Sherb. toxins evaluated in cereal grain with Trichoderma harzianum. Journal of Plant Protection Research 51 (2): 134-139.

Xie G., Tan S., Yu L. 2012. Morphological and molecular identification of pathogenic fungal of post-harvest tomato fruit during storage. African Journal of Microbiology Research 6 (22): 4805-4809.

Zainudin N.A.I.M., Perumal N. 2015. Mycotoxins production by Fusarium and Aspergillus species isolated from cornmeal. International Journal of Agriculture and Biology 17 (3): 440-448. 